\title{
An interactive graph visualization for handling cooperative design activity context
}

\author{
Gilles Halin \\ Nancy University, \\ National Superior School of Architecture of Nancy \\ UMR MAP-CRAI, rue Bastien Lepage, Nancy, France \\ halin@crai.archi.fr
}

\begin{abstract}
The context of a collective activity is characterized by all information produced during the activity. The comprehension of this context by each actor is essential for the success of the activity. The mediatization of this context in software requires the definition of its data representation, but also its visualization representation in a human machine interface. We propose here a network representation of this context and an interactive visualization of graph allowing each actor to have simultaneously a global and local perception of the context, to handle it with adapted navigation functions and to modify it with intuitive edition functions. These representation and visualization forms were implemented in the framework of an architectural design activity.
\end{abstract}

Keywords: CSCW, Collaborative Design, Visualisation, Man-Machine Interface, Model approach

\section{Introduction}

A collective activity generates a large quantity of very varied information. This information produced during the activity characterizes the "context" [1] of the activity. The comprehension of this context by each actor is essential for the success of the activity. The usual visualization of information in a data-processing tool dedicated to the document exploitation rests on a hierarchical representation (a folder, a sub-older, a document). When the tool is specialized in the management of a collective activity, the visual organization, inside the spaces of the activity: production, coordination, communication [2] is similar. Thus a hierarchical organization of the produced documents stays in the production space, a hierarchical presentation of the tasks (phase, task, sub-task) is present in the coordination space and a list of the exchanges (emails) placed in folders relative to the organization of the group structures the communication space. These views on the context, though useful to understand the progress of each space, are only partial and localised views of the whole of the activity. The use of these hierarchical forms of visualization shared by each actor does not favour the global perception of the context and its comprehension by a given actor. In order to understand the visualized context, each actor must provide an important cognitive effort to put in relation, according to his role in the activity, all the relevant elements presented in the various hierarchical views (actor, document, activity, exchange, event,...).

Indeed, the context of a collective activity has not a hierarchical structure but rather a network one. Each element of the activity (actor, document, activity, exchange,...) is in relation or in interaction with other elements: for example a document is produced by an actor who sent a message to another actor to carry out a given task.

We present in this article a graphic interface which, starting from an interactive visualization of a graph (or network), allows at the same time a local, global and personalized perception and management of a collective activity context. This visualization uses a network type representation of the activity context, modelled in a hyperdocument form, where each element and relation is described.

This interface was implemented to visualize the context of an activity of co-operative architectural design. The first part of this article presents the representation of the context used by the visualization. The second part describes the contributions of the interactive visualization of graph. Finally the last part presents the realized tool used for visualizing the context of a cooperative activity of architectural design.

\section{Cooperative context representation}

The creative aspect of the co-operative design activity requires a great implication of the actors [3]. One of the means proposed to reinforce the creative and operational capacity of this type of organization is the creation of a conscience of group (awareness) by an equitable diffusion of information relating to the evolution of project [4].

The context of an activity of collective design and more particularly that of the architectural co-operative design is characterized by a set of hierarchical and egalitarian relations between actors [5]. Each element of 
the project has a specific environment where it generates interactions. For example, an actor writes, modifies, annotates, and consults documents; it takes part in activities and exchanges some information with other actors who are also implied in the project. The model we propose is the representation of these interactions in form of relations between the various elements of the project. The definition of this model [6] uses an approach guided by the models [7] like those proposed in the standard MOF [8] of the OMG (Object Management Group).

The application of the meta-model of the context of co-operation (MMCCOO) to the architectural design was carried out by an instanciation of the various classes present in the meta-model (Actor, Activity, Document, Relation). This instanciation was realized with the instructions indicated in the MOP French law which defines the missions of each actor who participates in the mastery of public building work. Figure 1 shows an extract of this MMCCOO metamodel instanciation, which produces the definition of a model of the context of co-operation in conformity with the MOP law (MCCOO). This model allows the generation of a particular context as in the example the design of a school.

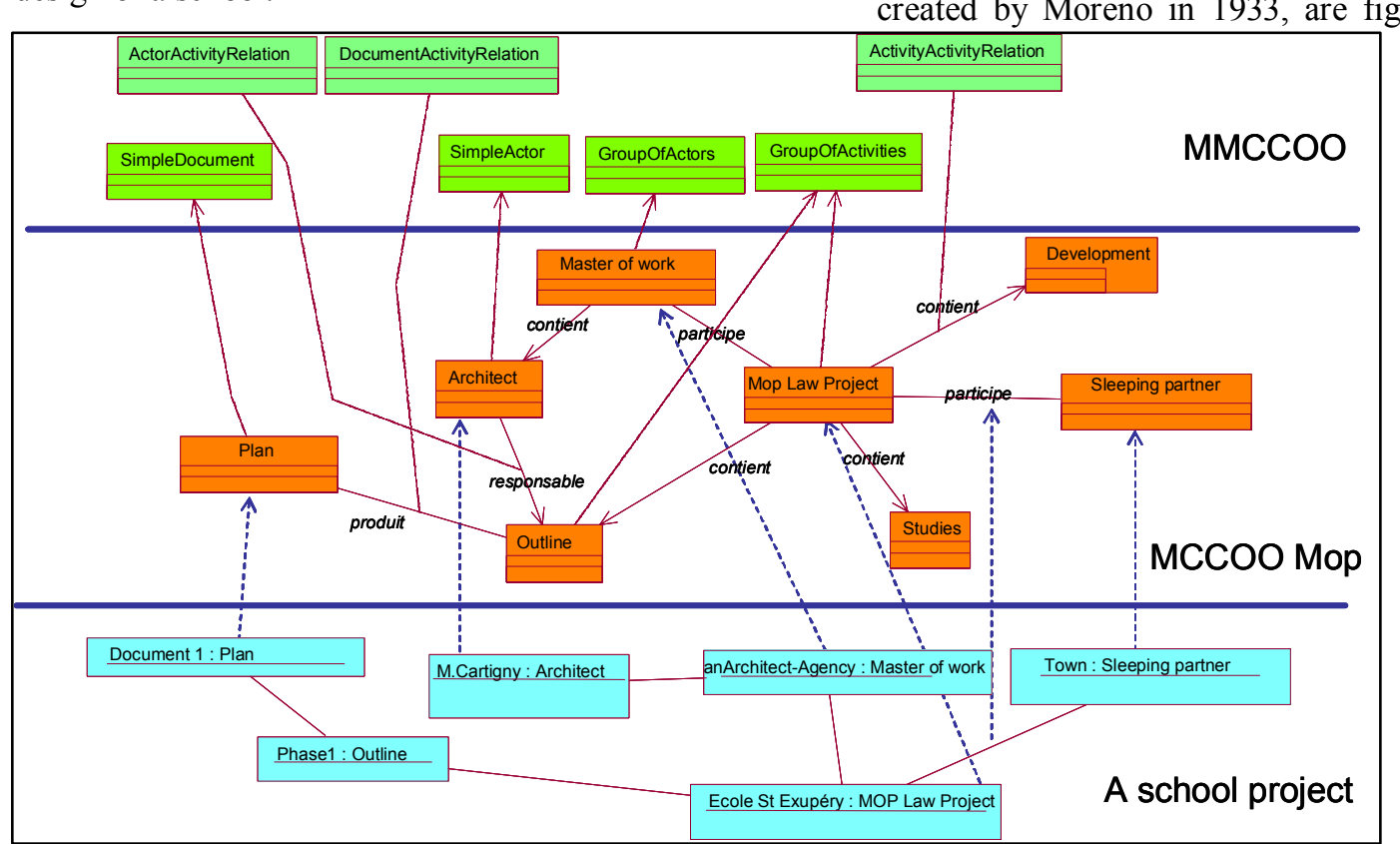

Figure 1. Level of the modelling approach

From the point of view of the information organization, the representation of a context of cooperation obtained by this model approach (cf Figure 1) has the structure of a network assimilate to a hyperdocument. Hypermedia technologies are useful for data representation and management (visualization, navigation) but also for the user-adapted interactions they propose [9]. We formulate the first assumption that more the project representation is near to the organization perceived by the members of this project, more this vision of the project will be accessible to the users.

\section{Graphic and interactive visualization}

The visualization of a mass of information by a dataprocessing tool does not depend simply on the structure of data which describes it, but also on activity of the user. Indeed, when visualization becomes interactive, it stimulates the cognitive activity of user [10] by amplifying his capacities of perception. Thus these are the information usage and the type of data which condition the choice of the representation and the associated interactivity.

From these observations we express the second assumption that it is possible to assimilate the representation of the organization and the evolution of a collective activity to the visualization of an adaptive and interactive graph.

\subsection{Graph visualisation}

The use of graphs for visualizing the interactions inside a group is not a recent approach. The sociograms, created by Moreno in 1933, are figures in which the social entities are represented by points and the interactions between the pairs of people by lines [11]. They allow the analysis of the social rapports inside a group by representing either the frequency of the exchanges (thickness of link), or the type of the exchanges (form of the link) or the type of the social entities taking part in the exchanges (form of the points of the graph).

The visualization of information in the graph form is also the support of the presentation of the results of an information retrieval. It allows at first a fast exploration of the results by a global perception, then the analysis of relations and access paths in relevant information.[12]. The Kartoo (www.kartoo.com) research meta-engine and the Touchgraph GoogleBrowser tool (www.touchgraph.com) illustrate this approach by the construction of thematic maps or 
graphs obtained by a textual analysis of the found Web references.

The techniques of construction and representation of graph differ essentially by the volume and the use of information presented. When volume is important, the graph will be exclusively made up of identical points and simple links. The objective of visualization is then to allow a global perception of the information organization, either to extract some from the general characteristics of volume, or to identify a relevant part of the graph on which the attention then will be focused. [13].

On the other hand when the visualized graph has a restricted number of information allowing the identification of the nodes and the links presented, it becomes interesting to propose a form of visualization where the nodes are represented by icons supporting the identification of their function in the graph, and where the links have a graphical representation adapted to the meaning of the relation they materialize. This type of graph has two forms of presentations: a static one where the nodes have a fixed position and a dynamic one where the graph is controlled by a force system and the node position is determined by the obtaining of a state of balance of system [14].

\subsection{Interactive visualisation}

When a graph visualization is interactive it becomes a powerful tool for navigation after a short period of adaptation. An incremental navigation by progressive deployment or selective masking of nodes or links is the form of navigation generally proposed. Other functions of browsing or presentation of the graph are associated with this form of navigation: the zoom function is used to focus on a part of the graph; the locality function makes it possible to visualize a more or less high number of nodes near a selected node; the rotation of the graph and the node moving allow the user to organize personally the graph fitting; the filters give the possibility to select the types of nodes or links in order to simplify the readability of the graph; the search for nodes; a historic of navigation; the obtaining of further information by passing the cursor of the mouse on a node or a link.

The Brain tool (www.thebrain.com), used in management tools of knowledge, proposes a static presentation of the nodes associated with a hierarchical browsing of an arborescent structure of graph (father nodes, sons and brothers, close relations). The dynamic presentation is that used in the Thinkmap (www.thinkmap.com) and Touchgraph tools (www.touchgraph.com). The first is a commercial tool implemented in the thesaurus visualization and browsing. It proposes an incremental navigation with historic, an information complement (definition), a graph fitting and a set of filters on the types of words (verb, adjective, adverb,...). The second is a free software proposing the visualization of an interactive graph described in a XML file. He is available in the form of a Java applet or a Java application which was adapted to several contexts of use going from the presentation of the results of a research on the Google engine (cited above) to the presentation and the edition of a wiki. The proposed functions of browsing are based on an incremental navigation with locality, zoom and information complement. Some functions of graph edition are present: addition, modification and suppression of nodes and links.

This is the last tool that we chose to adapt for visualizing, navigating and editing the hyperdocument which describe the co-operative context of a collective activity.

\section{Towards an interactive cooperative context manipulation}

The Hyperdocument obtained from a model of context of co-operation (MCCOO) has a network structure which can be visualized in the shape of a graph where each node is either an actor, a document, or an activity and where each arc is a link between these various elements of the context. The Bat' Map interface, developed from the TouchGraph tool, implements this type of visualization (cf. Figure 2).

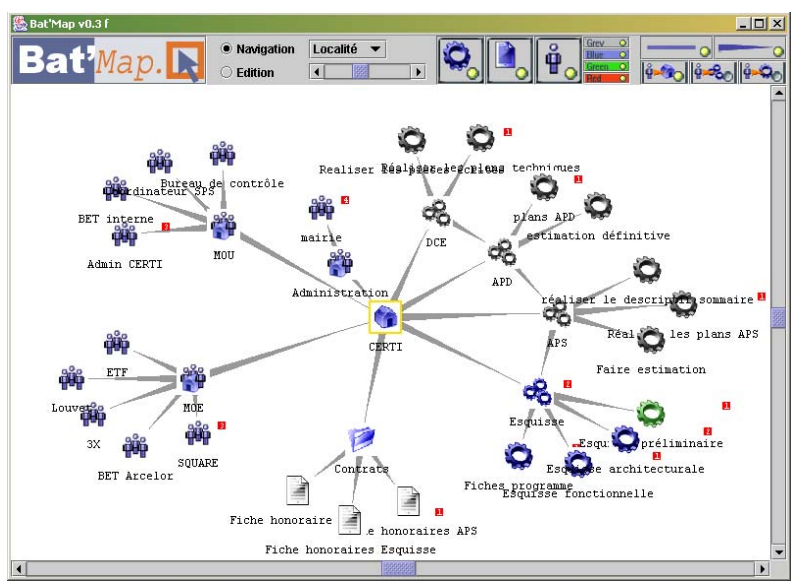

Figure 2. the Bat'Map interface

\subsection{Visualization of the context}

In this form of visualization, the quantity of information presented takes an important part in the perception and the understanding of the user. To facilitate perception, the graph is presented with a locality of three starting from the node "project". A not deployed node has a red label which indicates the remaining number of levels to deploy. The use of the icons allows the user to quickly identify the various types of node which correspond to the basic concepts of the model (group/actor, file/document, activity/group of activities). The colour of the icons informs about the progress report of the elements of the project. Thus a "not started" task is coloured in gray, a task "in 
progress" in blue, a "finished" task in green, and finally a task which has a problem in red (cf. Figure 5). The nodes are connected by one-way or bidirectional links. The one-way link is used to represent, the hierarchy between the nodes, like a file and a document or a group and an individual person, but also to materialize the nonsymmetrical relations, like the relation "refers" between two documents. The symmetrical relations between two types of nodes are represented by the bidirectional bonds, like the relation "collaborates with" between two actors.

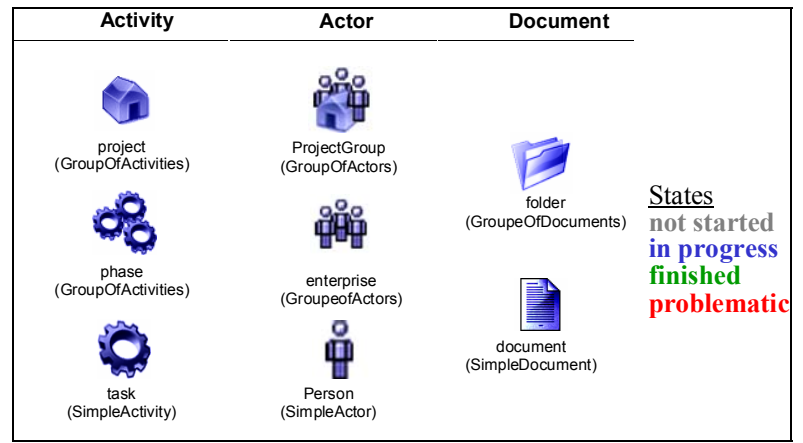

Figure 3. Graphic representation of nodes

All these choices of graphic representation offer a global perception of the context of co-operation and a comprehension of its dynamic aspect by answering with a unique view to the essential questions that any actor taking part in an activity of design can formulate: which are the tasks in progress? In which task I have to participate? Who collaborates with me in this task? Which are the documents to be produced? Who already worked in this task? ... The obtaining of understandable answers to these questions generates conscience of group which is essential to any collective activity.

\subsection{Browsing the context}

The proposed navigation forms have a role of increasing the perception and the comprehension of the context without generating disorder or cognitive confusion. In the Bat'Map interface, various possibilities are offered to the user to navigate through the graph and to interact with it. First, all the traditional functions of graph browsing are present: a geometrical zoom, the rotation of the graph, the nodes organization fitting, the locality and incremental navigation.

With these forms of navigation, a system of filters (cf. Figure 4) additive/subtractive has been added giving the possibility to the user to select at the same time the types (nodes or relations) and the states (not started, in progress, finished, problematic) of information that he wishes to visualize. Thus, a user, wishing to see the not yet started tasks and the actors implied in these tasks, will activate the filters "Actor", "Activity" and the state "gray". The filters on the links will enable him to refine its visualization by specifying the type of relation that it wishes to see. For example by filtering only the one-way relations, visualization will be centered on the structure of groups and activities.

\subsection{Editing the context}

The Bat' Map interface makes it possible to edit graphically the context of co-operation. Thus it allows each actor to act on this context in a controlled way. By activating the edition mode, the user-actor can create a new node by simple "drag and drop". He can modify information relating to an existing node by editing the associated properties. For example, according to his role in the collective activity, a user-actor can add a new activity to be carried out with a group of actor (cf. Figure 5). Thus the user-actor adds visually new information and puts it directly in relation to the context of the activity in which it takes part.

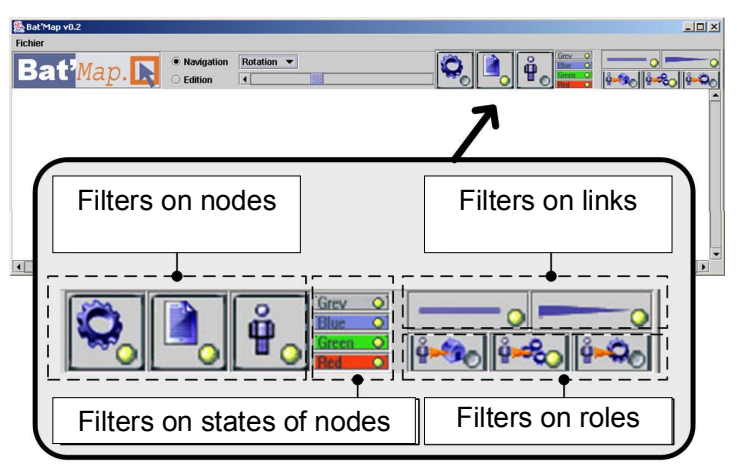

Figure 4. The filters available for browsing

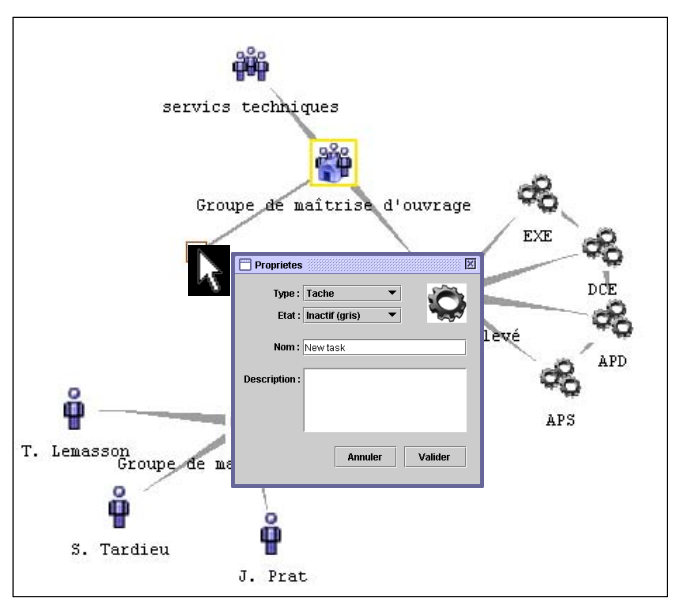

Figure 5. An example of graph editing

\subsection{Experiment}

The implementation of this contextual visualization was realized in the "Bat'Group" co-operative platform which was used as support to experimentation. Two forms of interfaces were implemented for the access to the Bat' Group platform: Bat' Map we have just presented and Bat'Classic (cf. Figure 6) a traditional HTML interface which proposes a hierarchical visualization of the elements of the collective activity. 
A scenario of co-operative design was implemented by two groups of students. The first group developed the scenario by using the Bat'Classic interface whereas the second used the Bat' Map interface. The experiment highlighted the interest of the interactive visualization of graph within the framework of a "simulated" collective activity. Indeed, the actor-students using the graphic interface realize the scenario more quickly than those which handled the traditional interface. In each new capture of scenario interaction, the students of Bat'Classic encountered some difficulties of determining the exact place (context) in which to put new information (actor, task or document). On the other hand, the users of Bat' Map were surprised by the facility it offered for the addition of new information in the activity: creation of a node and a link in the suitable context. This experiment highlighted that this new form of visualization is apprehensible by actor-students and it allows more effectively than a traditional visualization to perceive, understand and modify the context of a cooperative activity.

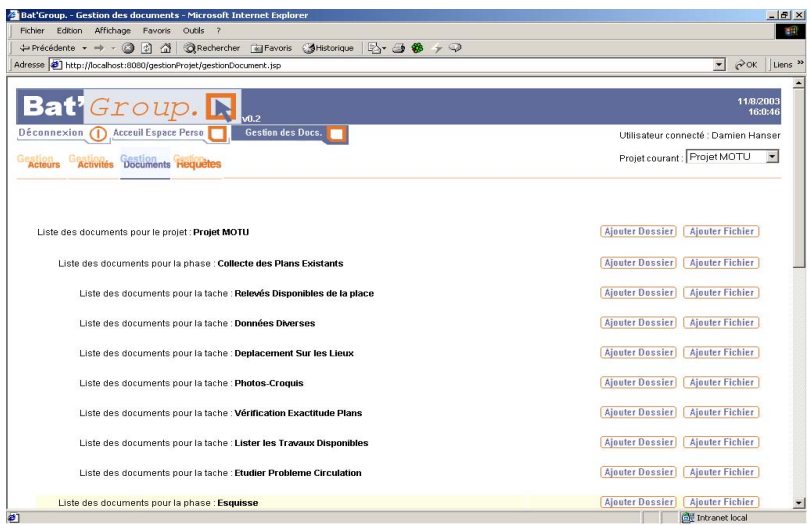

Figure 6. The Bat'Classic interface of the Bat'Group platform

\section{Conclusion}

Co-operative work inside an activity of design requires a good representation and visualization of the context in order to support its comprehension by each actor and to preserve the autonomy and the potential of action of each one and thus stimulate creation and the innovation. The traditional forms of visualization are based on a hierarchical representation of the context of co-operation. This sort of representation is not adaptable to a global perception of the activity context which is necessary for the located action of each actor. The interactive visualization of graph, that we described here, represents an interesting alternative since it proposes a visual representation near of the relational structure of the context of co-operation. The forms of proposed navigation and edition make it possible to each actor to identify his sphere of activity (localization in the graph) and then to act directly on this context by simple and intuitive interactions (drag and drop).
This new form of visualization uses a context representation defined with the principles of the models driven engineering by the (MDE) which enabled us to identify the concepts of a collective activity of design that we used to describe the co-operative context of the architectural design. This open framework of modeling gives us the possibility to define new forms of visualization adapted to different collective activity contexts. This is a way we follow with the Bati'views project in order to propose an adapted multivisualization of the building-work activity context [15]

\section{References}

[1] P. Brézillon, "Role of context in social networks," presented at 18th Florida Artificial Intelligence Research Society (FLAIRS2005), Clearwater Beach, Florida, 2005.

[2] G. Calvary, J. Coutaz, and L. Nigay, "From Single-User Architectural Design to PAC*: a Generic Software Architecture Model for CSCW," presented at CHI'97, 1997.

[3] T. Kvan, "Collaborative design : what is it ?," Automation in Construction, vol. 9, pp. 409-415, 2000.

[4] P. Dourish and V. Bellotti, "Awareness and Coordination in Shared Workspaces," presented at CSCW (Computer Supported Cooperative Work), Toronto, Ontario, Canada, 1992.

[5] P. Bernoux, La sociologie des organisations, 3ème édition ed. Paris Seuil (Points), 1990.

[6] G. Halin, D. Hanser, and J.-C. Bignon, "User adaptive visualization of cooperative architectural design," International Journal of Architectural computing, vol. 02, 2004.

[7] C. Atkinson and T. Kühne, "Model-Driven Development: A Metamodeling foundation," IEEE Software, vol. 20, pp. 3641, 2003.

[8] OMG, "Meta Object Facility (MOF) Specification," Object Management Group, http://www.omg.org/, OMG Document formal 2000-04-03 2000.

[9] P. Brusilovsky, "Adaptive hypermedia," User modelling and User-Adapted Interaction, vol. 11, pp. 87-110, 2001.

[10] B. Shneiderman and C. Plaisant, Designing the User Interface. Boston, USA: Addison Wesley, 2005.

[11] J. Scott, Social Network Analysis, a handbook, ed. London: Sage Publications, 2000.

[12] S. K. CARD, "Visualizing retrieved information : A survey : Computer graphics and visualization in the global information infrastructure," IEEE computer graphics and applications vol. 16, pp. 62-67, 1996.

[13] I. Herman, G. Malançon, and S. Marshall, "Graph Visualization and Navigation in Information Visualization : a Survey," IEEE Transactions on Visualization and Computer Graphics, vol. 6, 2000.

[14] M. Hascöet and M. Beaudoin-Lafon, "Hypermedia exploration with interactive dynamic maps," International Journal on Human Computer Studies, pp. 441-464, 1995.

[15] S. Kubicki, J. C. Bignon, and G. Halin, "Building construction coordination by an adaptive representation of the cooperation context," in Joint International Conference on Computing and Decision Making in Civil and Building Engineering. Montreal, Canada, 2006. 